\title{
Does a small size needle puncture cause intervertebral disc changes? ${ }^{1}$
}

\author{
Ana Carolina Issy', Vitor Castania" ${ }^{\mathrm{II}}$ Joao Walter Silveira ${ }^{\mathrm{III}}$, Marcello Henrique Nogueira-Barbosa ${ }^{\mathrm{IV}}$, Carlos Ernesto Garrido \\ Salmonv, Elaine Del-Bel ${ }^{\mathrm{VI}}$, Helton Luis Aparecido Defino ${ }^{\mathrm{VII}}$
}

DOI: http://dx.doi.org/10.1590/S0102-865020150080000009

IPhD, Postdoctoral, Department of Morphology, Physiology and Basic Pathology, Dental School of Ribeirao Preto, Universidade de São Paulo (USP); Neuroscience Research Nuclei (NAPNA), Brazil. Conception and design of the study, interpretation of data, manuscript writing, critical revision. IIPhD, Postdoctoral, Department of Biomechanics, Medicine and Rehabilitation of Locomotor System, Medical School of Ribeirao Preto (FMRP), USP, Brazil. Analysis of data, microscopic examinations, technical procedures.

IIIPhD, Postdoctoral, Department of Biomechanics, Medicine and Rehabilitation of Locomotor System, FMRP, USP, Ribeirao Preto-SP, Brazil. Design of the study, statistical analysis.

${ }^{\text {IV }} \mathrm{PhD}$, Associate Professor, Department of Internal Medicine, FMRP, USP, Ribeirao Preto-SP, Brazil. Analysis of data.

${ }^{\mathrm{v}} \mathrm{PhD}$, Associate Professor, Department of Physics, Faculty of Philosophy and Science Ribeirao Preto, USP, Brazil. Analysis of data.

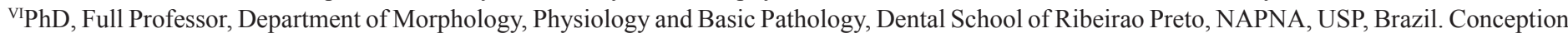
and design of the study, manuscript writing, critical revision.

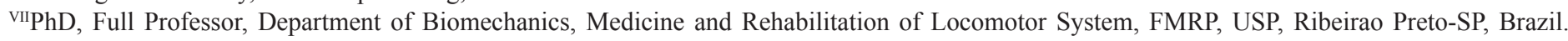
Conception and design of the study, manuscript writing, critical revision.

\section{ABSTRACT}

PURPOSE: Small size needles have been regularly used for intradiscal injection of innocuous/potential therapeutic compounds in experimental conditions, but also in clinic procedures, such as discography. Our aim was to investigate if a 30-gauge needle could trigger observable changes on intact intervertebral discs. We compared these effects to those induced by a large size needle (21-gauge), a well-known intervertebral disc degenerative model based on needle puncture.

METHODS: Coccygeal intervertebral discs (Co8-9) of adult male Wistar rats were punctured with a 21-gauge needle, while the coccygeal levels Co7-8 and Co9-10 remained intact. The 30-gauge needle was used to inject a safe volume of saline ( $2 \mu 1)$ on both intact (Co9-10) and punctured (Co8-9) discs. MRI and histological score were performed at 2, 15 and 42 days after procedure.

RESULTS: MRI analyses revealed significant reduction on signal intensity of 21 -gauge punctured discs. Intact discs which received a saline injection through a 30-gauge needle also revealed significant alterations in the MRI signal when compared with control discs. No histological changes were observed in the intact saline injected discs at any time analyzed.

CONCLUSION: Since significant intervertebral image changes were observed with a 30-gauge needle, cautious interpretation of the pharmacological inoculation findings is required.

Key words: Intervertebral Disc Degeneration. Models, Animal. Rats, Wistar. 


\section{Introduction}

The socioeconomic impact of low back pain associated with disc degeneration has required considerable research to propose treatment options. Potential therapies may be based on injections of anti-inflammatory/analgesic drugs or bioactive agents (ex. growth factors or cells), and the development of carrier systems for the delivery and the increase of these active compounds effects is of great interest ${ }^{1}$.

Animal models of intervertebral disc degeneration play an important role to test novel therapeutic strategies. Among others, the degenerative process induced by needle puncture has gained popularity because it is uncomplicated, reproducible, and cost-effective, and requires a short time to produce the disc degeneration ${ }^{2-4}$. While human disc degeneration is a complex and multifactorial process that occurs over decades, the limited anular injury induced by needle puncture is a potential initiator of disc degeneration ${ }^{2,3}$. Usually, needles with 18-22 gauge can produce morphologically and biochemically altered features similar to many of those found in human degenerative disc disease ${ }^{3-5}$. Conversely, small size needles, equal or superior to 29-gauge, are necessary to perform intradiscal injection in experimental conditions, which represents a controversial procedure in experimental practice, but also in clinical situation ${ }^{6-8}$

Recently, the discography has been linked to accelerated degeneration in human lumbar discs ${ }^{8}$. Opposing, in mice, the caudal disc puncture with a small gauge needle (29-gauge) did not initiate degenerative changes in disc structure and composition ${ }^{4}$. Since our group and others, have regularly used the 30-gauge needle for intradiscal injection of innocuous or potential therapeutic compounds, our aim was to investigate if this procedure could trigger observable changes on intervertebral disc. We used the magnetic resonance imaging (MRI) and histological analysis, the same tools usually selected to determine the effects of potential therapeutic agents.

\section{Methods}

Ethics Committee for Animal Experimentation of the Universidade de São Paulo, Campus Ribeirao Preto, Brazil, approved this research (CETEA, protocol n 016/2012). Male Wistar rats (300-350g; $n=14)$ were used. Animals were housed in groups of 3-4 and kept at temperature of $23{ }^{\circ} \mathrm{C}( \pm 1)$ with a 12-h light-dark cycle. Food and drinking water were available ad libitum.
Animals were anesthetized in an aseptic setting with ketamine $(50 \mathrm{mg} / \mathrm{kg})$ and xylazine $(5 \mathrm{mg} / \mathrm{kg})$ association. The coccygeal intervertebral spaces Co7-8, Co8-9 and Co9-10 were selected for the study. Disc degeneration was induced at the coccygeal disc Co8-9 using a 21-gauge needle according to the technique previously described ${ }^{6}$. The coccygeal discs Co7-8 and Co9-10 remained intact. In both coccygeal discs, Co8-9 (punctured with 21-gauge needle), and Co9-10 (intact), a 30 gauge needle was used for intradiscal injection of saline $(2 \mu \mathrm{l})$. The saline volume was selected based on previous observations that no significant changes are observed in the rat-tail discs injected with this volume ${ }^{9}$. The injection was performed with needle attached to a microsyringe (Hamilton $10 \mu \mathrm{l}$, USA) with an infusion pump interposed by a polyethylene catheter (PE10) with the velocity of $1 \mu \mathrm{l} / \mathrm{min}$. Needle stayed at intervertebral disc for an additional $3 \mathrm{~min}$ period to prevent reflux similar to the most pharmacological studies.

The MRI was conducted under general anesthesia. Images were acquired in a 3.0 T MR scanner (Philips ${ }^{\circledR}$, Achieva, The Netherlands) using a dedicated coil for small animal, as described previously described ${ }^{6}$. The disc signal intensity was estimated from the T2-weighted image (Echo time $=80 \mathrm{~ms}$ ) as an indirect measure of disc conditions and its water content. Intervertebral discs from $\mathrm{Co} 7-8$ to $\mathrm{Co} 9-10$, from each animal, were analyzed using the software Image $J^{\circledR}$. In order to evaluate the entire disc, five sequential sagittal images of intervertebral discs were considered, and the mean pixel intensity value was quantified.

Animals were euthanized with an excessive dose of ketamine/xylazine at $2(n=5), 15(n=5)$ or $42(n=4)$ days after intradiscal injection of saline with a 30 -gauge needle. The whole disc with adjacent vertebrae of selected coccygeal segments Co7-8, Co8-9 and Co9-10 were removed and dissected. Tissue was fixed in paraformaldehyde 4\%, pH 7.4, during 24 hours and decalcified in ETDA solution (hydrochloric acid 12\%, EDTA 0.07\%, sodium tartrate $0.014 \%$, potassium and sodium tartrate $0.8 \%$ and water) for 24 hours. After decalcification, coccygeal vertebrae and adjacent discs were sectioned midsagittally parallel to the direction of puncture with a scalpel. Midsagittal sections $(5 \mu \mathrm{m})$ were staining with Hematoxylin and eosin and were histological graded in blinded fashion using the definition established by Norcross and et al. ${ }^{10}$, under a light microscope (Leica ${ }^{\circledR}$, Germany) at 10X magnification. A grade score ranged from 1 (severe degenerated disc) to 5 (normal disc) was assigned separately to both annulus fibrosus and nucleus pulposus (Table 1 - modified from Silveira et al. ${ }^{6}$ ). 
TABLE 1 - Histological grading score.
Nucleus pulposus (NP)
5 Large, bulging central cavity with abundant NP material; $>2$ /3 IVD height; smooth borders with minimal disruption; 4 Slightly reduced central cavity size with some NP material present; $>1 / 3$ IVD height and $<2 / 3$ IVD height; minimal border disruption may be present;
3 Markedly reduced and disrupted cavity with minimal NP material and compartmentalization; total cavity $>1 / 3$ IVD height and $<2 / 3$ IVD height;
2 Severe disruption of NP with minimal cavity; total cavity $<1 / 3$ IVD height but $>0$; consists only of a few small pockets lined by NP-like cells;
1 Complete obliteration of cavity with no NP-lined pockets.

\section{Annulus fibrosus (AF)}

5 Discrete, well-opposed lamellae bulging outward with no infolding; minimal preparation defect with "simple radial clefting"*;

4 Discrete lamellae, less well-opposed; minimal infolding may be present; fibers remain well-organized, but with "complex radial clefting"**;

3 Moderate to severe infolding of discrete, relatively well-opposed lamellae; moderate fragmentation of lamellae; AF fibers remain well organized;

2 Severe infolding and distortion of poorly opposed lamellae; severe fragmentation of lamellae; small regions of disorganized fibrous material replacing central lamellae;

1 Severe infolding, distortion, and fragmentation of lamellae; extensive amount of disorganized fibrous material replacing central lamellae.

*Defined as the presence of radial gaps between AF lamellae with minimal fragmentation. **Defined as the presence of radial, transverse, and/or oblique gaps in the lamellae with significant fragmentation. This scale mainly scores the disruption of nucleus pulposus central cavity and cellularity, and the collagen fiber orientation of annulus fibrosus ${ }^{10}$.

The MRI data (mean \pm SEM; $n=5-7$ ) was analyzed by one-way ANOVA followed by the Newman-Keuls Multiple Comparison Test. The histological scores analyses were evaluated by the non-parametric Kruskal-Wallis test followed by Mann-Whitney for individual differences. Data was represented as the median value. In all cases, the level of significance was set at $\mathrm{p}<0.05$.

\section{RESULTS}

$M R I$

T2 images values correlate with the water content of intervertebral disc tissue and thereby allow the indirect measurement of nucleus pulposus hydration. Significant decreased signal intensity was observed at 2, 15 and 42 days after the 21-gauge puncture procedure (Co8-9) in comparison with control (Co7-8) discs. Also, intervertebral discs which received a saline injection with a 30-gauge needle (Co9-10) demonstrated significant reduction of MRI signal intensity compared to the control discs after $2(\mathrm{p}=0.001), 15(\mathrm{p}=0.001)$, and 42 days $(p=0.001)$. The loss of signal on T2-weighted images was higher in induced degenerated discs (Co8-9) at all evaluation periods (Figure 1).
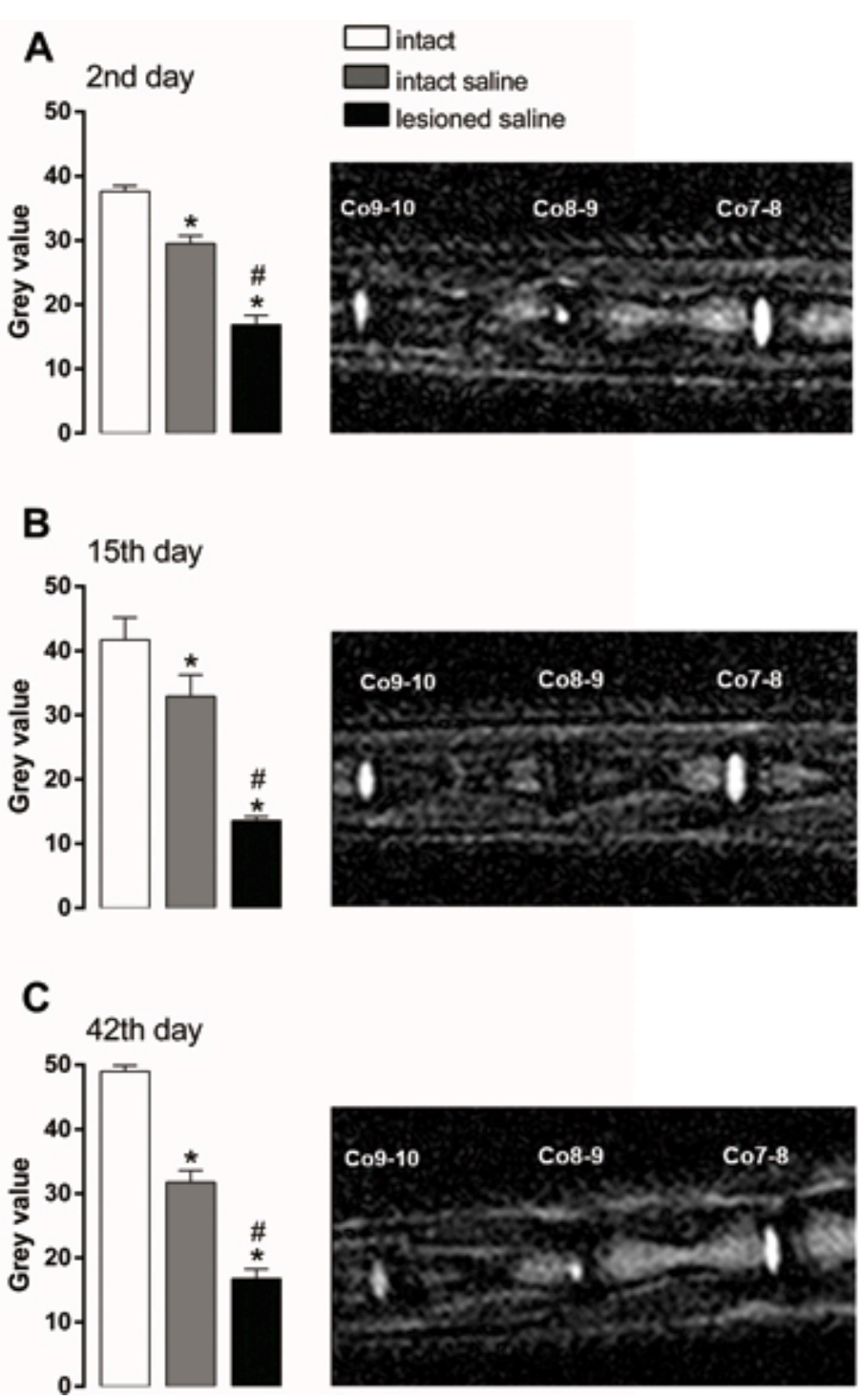

FIGURE 1 - MRI signal intensity. Data are described as the mean value of a grey scale (left panel). A representative T2-weighted MRI of rat-tail segments containing three intervertebral discs (Co7-8/8-9/9-10) is shown at right panel. Both the lesioned (21-gauge needle puncture; Co8-9) and the intact discs (Co9-10) which received saline $(2 \mu \mathrm{l})$ with a 30 gauge needle showed a significant decrease of MRI signal intensity compared to intact discs (intact control; Co7-8). These effects were similar at 2 (A), 15 (B) or 42 (C) days after the procedure. ${ }^{*} p<0.05$ compared to intact control; \# $\mathrm{p}<0.05$ compared to intact saline. 


\section{Histological analyses}

No histological changes were observed when the vehicle was injected into the intact disc despite the fact that a rigorous analysis revealed some qualitative alteration (Figure 2). Established histological changes were found in the intervertebral disc after 21-gauge needle puncture. Disc sections showed an annulus fibrosus with lamellar disorganization/fragmentation. Nucleus pulposus shows complete obliteration of its cavity with fibrous material. Also, there is a significant decrease of the intervertebral disc height. Significant worse histological score was found in the 21-gauge punctured discs both at nucleus pulposus (NP) and annulus fibrosus (AF) at 2 [NP; $\mathrm{p}=0.004 ; \mathrm{AF} ; \mathrm{p}=0.006], 15$ [NP; $\mathrm{p}=0.003 ; \mathrm{AF} ; \mathrm{p}=0.005]$, and 42 days $[\mathrm{NP} ; \mathrm{p}=0.037 ; \mathrm{AF} ; \mathrm{p}=0.022]$ after lesion. Table 2 presents temporal analysis of histological scores concerning changes of nucleus pulposus and annulus fibrosus at different evaluation period. The histological analysis was performed separated in the nucleus pulposus and annulus fibrosus in three different conditions. Histologic appearance of degenerative changes was found in the lesioned discs punctured with a 21-gauge needle. Intact discs injected or not with saline do not presented histological modifications.

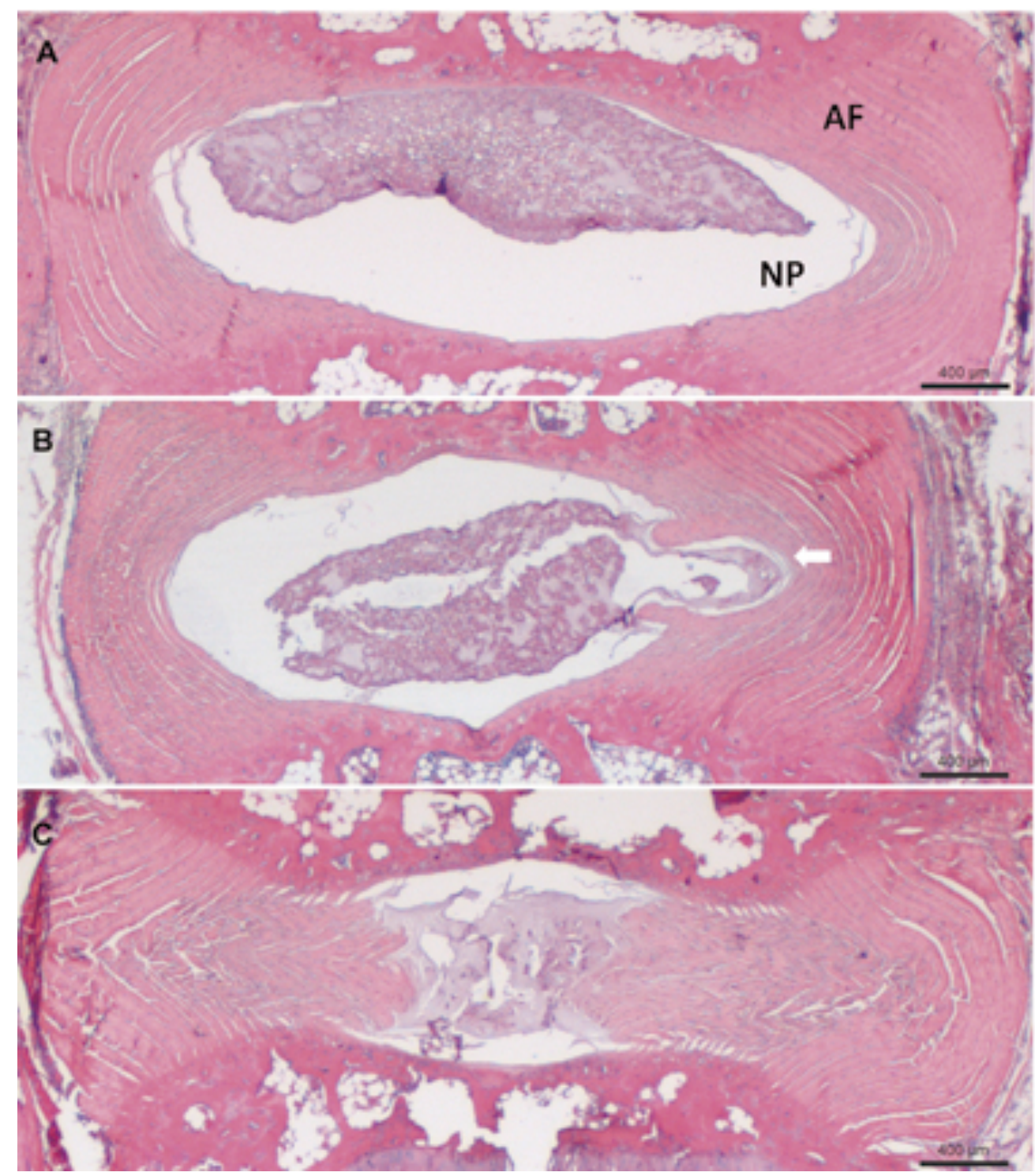

FIGURE 2 - Representative histological sections from intervertebral disc. (A) Intact disc showing well-organized fibers of annulus fibrosus (AF), and the nucleus pulposus (NP) comprising a significant disc area. (B) Intact disc at two days after saline injection with a 30-gauge needle showing the annulus fibrosus with concentric well-organized lamellas. Discrete alteration is revealed in the local of needle puncture (white narrow). The nucleus pulposus comprising a significant disc area, with exception of the local of a 30-gauge needle puncture. (C) Intervertebral disc at 42 days after a 21-gauge lesion and saline injection (30-gauge needle) showing an annulus fibrosus with marked lamellar disorganization, cavity obliteration and decreased height. No nucleus pulposus cells are observed (Sagittal sections-Hematoxylin and eosin, x5). 
TABLE 2 - Temporal analysis of histological score grade of tail rat intervertebral disc.

\begin{tabular}{lcccccc}
\hline Time (days) & $\begin{array}{c}2 \\
(\mathrm{n}=5)\end{array}$ & & $\begin{array}{c}15 \\
(\mathrm{n}=5)\end{array}$ & & $\begin{array}{c}42 \\
(\mathrm{n}=4)\end{array}$ & \\
\hline Condition & $\mathbf{N P}$ & $\mathbf{A F}$ & $\mathbf{N P}$ & $\mathbf{A F}$ & $\mathbf{N P}$ & $\mathbf{A F}$ \\
\hline Intact control & 5 & 5 & 5 & 5 & 5 & 5 \\
Intact saline & 5 & 5 & 5 & 5 & 4.5 & 3.5 \\
Lesion saline & $2 *$ & $3 *$ & $1 *$ & $3 *$ & $2 *$ & $2 *$ \\
\hline
\end{tabular}

Data are reported as median value. ${ }^{*} \mathrm{p}<0.05$, compared to intact discs. NP, nucleus pulposus; $\mathrm{AF}$, annulus fibrosus.

\section{Discussion}

Our results revealed that small size needle puncture triggered deleterious effects in the rat-tail intervertebral discs. MRI evaluation indicated a significant loss of signal intensity at two days after disc puncture, which remained at least until 42 days after the procedure. These results confirmed and extended our previous data ${ }^{6}$ No histological changes were observed in the 30-gauge punctured discs, despite some discrete qualitative alterations in the local of annular puncture.

The needle puncture model has been frequently used to mimic some characters of human disc degeneration ${ }^{2-7}$. Generally, needle size is correlated with the severity of induced-intervertebral disc degeneration. Larger needle sizes (18-22-gauge) produce more disc deterioration when compared with smaller sizes (23-26-gauge $)^{4,5}$. On the other hand, small size needles are used to delivery disc therapies, or for some diagnostic procedures, and this practice has raised a lot of discussion. A clinical report of accelerated disc degeneration after discography highlighted the question about intradiscal injection procedures security ${ }^{8}$.

Elliot et $a l .{ }^{11}$ suggested that needle puncture inducing discal changes is dependent on the ratio of needle diameter to the disc height. In this case, disc changes were universal for the ratio over $40 \%$. The literature findings concerning the effect of small needle puncture on rat caudal discs are controversial. Our data corroborates the hypotheses that controversial results about the deleterious effects of small size needle puncture to the intervertebral disc health are also dependent of the accuracy of analyses tools. Despite changes in the T2 MRI indicate structural and matrix content alterations in the intervertebral, therefore indicating that 30-gauge needle may be harmful to the disc, histological analyses score did not show any discal modifications. Opposing, other authors, such as, Grunert et al. ${ }^{12}$ observed a good correlation between histological damage and injury suggested by the MRI.
Ultimately, our findings show the importance of the sensitivity of the valuation method in the results interpretation. How these changes correlate with loss of function needs further investigation.

\section{Conclusions}

Since significant intervertebral image changes were observed with a 30-gauge needle, cautious interpretation of the pharmacological inoculation findings is required.

\section{References}

1. Blanquer SB, Grijpma DW, Poot AA. Delivery systems for the treatment of degenerated intervertebral discs. Adv Drug Deliv Rev. 2015 Apr;84:172-87. doi: 10.1016/j.addr.2014.10.024.

2. Han B, Zhu K, Li FC, Xiao YX, Feng J, Shi ZL, Lin M, Wang J, Chen QX. A simple disc degeneration model induced by percutaneous needle puncture in the rat tail. Spine. 2008 Aug;33:1925-34. doi: 10.1097/BRS.0b013e31817c64a9.

3. Keorochana G, Johnson JS, Taghavi CE, Liao JC, Lee KB, Yoo JH, Ngo SS, Wang JC. The effect of needle size inducing degeneration in the rat caudal disc: evaluation using radiograph, magnetic resonance imaging, histology, and immunohistochemistry. Spine J. 2010 Nov;10(11):1014-23. doi: 10.1016/j.spinee.2010.08.013.

4. Martin JT, Gorth DJ, Beattie EE, Harfe BD, Smith LJ, Elliott DM. Needle puncture injury causes acute and long-term mechanical deficiency in a mouse model of intervertebral disc degeneration. J Orthop Res. 2013 Aug;31(8):1276-82. doi: 10.1002/jor.22355.

5. Zhang H, La Marca F, Hollister SJ, Goldstein SA, Lin CY. Developing consistently reproducible intervertebral disc degeneration at rat caudal spine by using needle puncture. J Neurosurg Spine 2009 Jun;10(6):522-30. doi: 10.3171/2009.2.SPINE08925.

6. Silveira JW, Issy AC, Castania VA, Salmon CE, Nogueira-Barbosa $\mathrm{MH}$, Guimarães FS, Defino HL, Del Bel E. protective effects of cannabidiol on lesion-induced intervertebral disc degeneration. PLoS One. 2014 Dec 17;9(12):e113161. doi: 10.1371.

7. Korecki CL, Costi JJ, Iatridis JC. Needle puncture injury affects intervertebral disc mechanics and biology in an organ culture model. Spine. 2008 Feb 1;33(3):235-41. doi: 10.1097/ BRS.0b013e3181624504.

8. Carragee EJ, Don AS, Hurwitz EL, Cuellar JM, Carrino JA, Herzog R. 2009 ISSLS Prize Winner: Does discography cause accelerated progression of degeneration changes in the lumbar disc: a ten-year matched cohort study. Spine (Phila Pa 1976). 2009 Oct;34(21):233845. doi: 10.1097/BRS.0b013e3181ab5432.

9. Mao HJ, Chen QX, Han B, Li FC, Feng J, Shi ZL, Lin M, Wang $\mathrm{J}$. The effect of injection volume on disc degeneration in a rat tail model. Spine (Phila Pa 1976). 2011 Jul;36(16):E1062-9. doi: 10.1097/BRS.0b013e3182027d42.

10. Norcross JP, Lester GE, Weinhold P, Dahners LE. An in vivo model of degenerative disc disease. J Orthop Res. 2003 Jan;21(1):183-8. doi: 10.1016/S0736-0266(02)00098-0.

11. Elliott DM, Yerramalli CS, Beckstein JC, Boxberger JI, Johannessen $\mathrm{W}$, Vresilovic EJ. The effect of relative needle diameter in puncture and sham injection animal models of degeneration. Spine. 2008 Mar;33(6):588-96. doi: 10.1097/BRS.0b013e318166e0a2.

12. Grunert P, Gebhard HH, Bowles RD, James AR, Potter HG, Macielak M, Hudson KD, Alimi M, Ballon DJ, Aronowitz E, Tsiouris AJ, 
Bonassar LJ, Härtl R. Tissue-engineered intervertebral discs: MRI results and histology in the rodent spine. J Neurosurg Spine. 2014 Apr;20(4):443-51. doi: 10.3171/2013.12.SPINE13112.

\section{Acknowledgement}

To Célia Aparecida da Silva for technical support.

\section{Correspondence:}

Prof. Helton Luis Aparecido Defino

Departamento de Biomecânica, Medicina e Reabilitação do Aparelho Locomotor

Faculdade de Medicina de Ribeirão Preto-USP

Avenida Bandeirantes, 3900, $11^{\circ}$ andar

14048-900 Ribeirão Preto-SP Brasil

Tel.: (55 16) 3315-2845

hladefin@fmrp.usp.br

Received: Apr 10, 2015

Review: June 13, 2015

Accepted: July 122015

Conflict of interest: none

Financial sources: CAPES, PNPD, FAPESP and CNPq

${ }^{1}$ Research performed at Laboratory of Molecular and Celular Neurophysiology, Department of Morphology, Physiology and Basic Pathology, Dental School of Ribeirao Preto, Universidade de São Paulo (USP), Brazil. 